\title{
Towards Extremely Sensitive Ultraviolet-Light Sensors Employing Photochromic Optical Microfiber
}

\author{
George Y. Chen ${ }^{1}$ and Zilong Wang ${ }^{2}$ \\ ${ }^{1}$ Laser Physics and Photonic Devices Laboratories, University of South Australia, Mawson Lakes Campus, Mawson Lakes, \\ SA 5095, Australia \\ ${ }^{2}$ Optoelectronics Research Centre, University of Southampton, Southampton, Hampshire SO17 1BJ, UK \\ Correspondence should be addressed to Zilong Wang; zwle09@orc.soton.ac.uk
}

Received 27 November 2014; Revised 30 March 2015; Accepted 20 April 2015

Academic Editor: Oleg Lupan

Copyright (c) 2015 G. Y. Chen and Z. Wang. This is an open access article distributed under the Creative Commons Attribution License, which permits unrestricted use, distribution, and reproduction in any medium, provided the original work is properly cited.

We propose an extremely responsive ultraviolet-light sensor $\left(-1.39 \times 10^{6} \mathrm{~dB} /\left(\mathrm{W} / \mathrm{cm}^{2}\right)\right)$ based on photochromic optical microfiber. A densely packed planar coil of ZBLAN optical microfiber is doped with photochromic dyes. Under ultraviolet radiation, the photochromic microfiber experiences temporary photodarkening, and the change in the transmission of the probe light provides a measure of the incident ultraviolet light. This novel design grants an enhancement in sensitivity $\left(3.13 \mathrm{nW} / \mathrm{cm}^{2}\right)$ by at least one order of magnitude compared to traditional electrical counterparts.

\section{Introduction}

Ultraviolet (UV) sensors are used in a wide range of applications, including fire detection, industrial manufacturing, biochemical research, light sources, and environmental and structural health monitoring. The spectral range of UV light lies between the X-ray (i.e., $10 \mathrm{~nm}$ wavelength and $124 \mathrm{eV}$ photon energy) and visible (i.e., $400 \mathrm{~nm}$ wavelength and $3.1 \mathrm{eV}$ photon energy) spectra. Typically, its spectral range is divided into four regions: UV-A for wavelength between 320 and $400 \mathrm{~nm}, \mathrm{UV}$-B for wavelengths between 280 and $320 \mathrm{~nm}$, UV-C for wavelength between 200 and $280 \mathrm{~nm}$, and far-UV for wavelength between 10 and $200 \mathrm{~nm}$.

The traditional electrical UV-light sensor [1], which consists of a sensitive photodiode that generates a photocurrent when exposed to UV light, is well established. The current is then converted into a voltage signal and amplified for easier detection. The sensing element of the traditional UVlight sensor is the photodiode, which is commonly based on silicon. Due to the low bandgap energy of silicon (i.e., $1.1 \mathrm{eV}$ ), expensive low-pass optical filters and phosphor coatings are required to block low-energy photons of longer wavelengths that are more abundant in a typical measurement environment. This limits the commercial appeal of such sensors. To overcome this hurdle, extensive research has been carried out on wide bandgap semiconductors, films, and 1-dimensional nanostructures as alternatives. Despite some promising results, their low reliability and low reproducibility prevent them from replacing traditional photodiodes in the short term.

Conventional fiber-optic UV sensors [2-4] employ functionalized coatings that emit luminescent light in the visible spectrum under UV radiation, which is subsequently detected by an inexpensive photodiode (i.e., no filter necessary). Their advantages are remote sensing capability, immunity to EMI, and higher robustness. However, the coating itself is an active medium that inherently exhibits power variations during ambient temperature drifts. This is a major disadvantage because it induces variations in the responsivity amongst other parameters such as UV sensitivity and accuracy.

Here, we propose a novel UV-A sensor based on photochromic optical microfiber (OM). It has the advantages of higher responsivity and higher power stability in addition to those already present in conventional fiber-optic UV sensors. 
The performance and feasibility of this sensor are theoretically modeled to demonstrate its potential for monitoring tanning rays from both natural and artificial sources, amongst other applications.

\section{Materials and Methods}

Under the influence of UV light, a photochromic molecule changes shape that leads to high absorption of visible light. This is a reversible effect because the molecule will revert back to its inactivated state when it is no longer being irradiated with UV light. The rate at which the photochromic dyes switch between the coloured and colourless states is dependent on the ambient temperature and the chemical composition of the dye.

The conventional way to reduce the diameter of an optical fiber is to taper it [5]. Optical fiber tapers are simply made by stretching a heated fiber. A structure is formed comprising a narrow stretched filament (i.e., the taper waist), each end of which is linked to an unstretched original fiber by a conical section (i.e., the taper transition). The uniform taper waist is known as the OM. For small core-cladding ratio starting fibers, mode guidance changes in the down-taper transition from a core-cladding to a cladding-surrounding interface. This is because the original core after tapering can be more than one order of magnitude smaller than the wavelength of light and thus cannot practically guide light. Additionally, the refractive index (RI) difference along the original cladding-surrounding interface can be up to 100 times larger than the RI difference along the original corecladding interface. Therefore, the original core has a marginal influence on the guiding properties and can be neglected. As a result, the shrunken original cladding is renamed as the core, with the surrounding medium renamed as the cladding. The advantages of using this starting fiber include larger evanescent field, interferometer and resonator possibilities, higher power stability, lower linear birefringence, and lower cost. For large core-cladding ratio starting fibers, the original core is still present after tapering. The original cladding is partially retained if the RI difference is small (e.g., <0.4) and fully retained if the RI difference is large (e.g., >0.4). The advantage of using this starting fiber is lower optical loss. If the transition is adiabatic, the optical loss of the transition regions is negligible. A small core-cladding ratio is better suited to the application of UV sensing. Therefore, the starting fiber is based on regular-sized optical fibers such as the telecom single-mode fiber (SMF-28).

The sensing mechanism is illustrated in Figure 1. Under UV-A radiation, the photochromic OM experiences temporary photodarkening (i.e., increased absorption throughout UV and visible wavelengths). Changes in the transmission of the visible light (i.e., $660 \mathrm{~nm}$ wavelength) generated by a laser diode are monitored by a power meter, which provides a measure of the UV intensity. The proposed experimental setup is shown in Figure 2.

The sensor can be built in five stages. The first stage involves the construction of a low-pass glass-disc enclosure

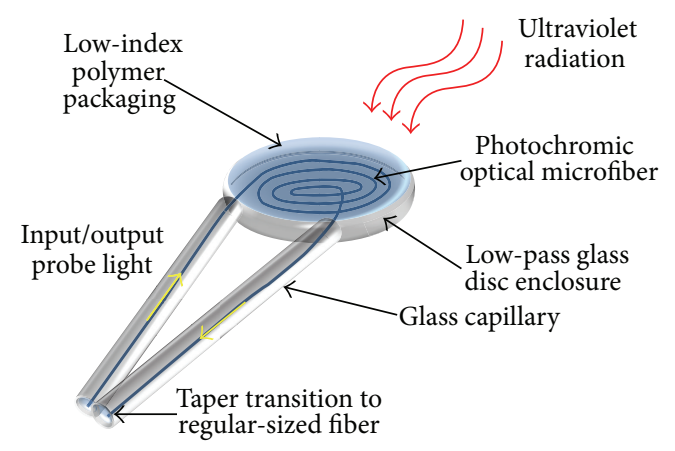

FIGURE 1: Schematic of the ultraviolet-light sensor.

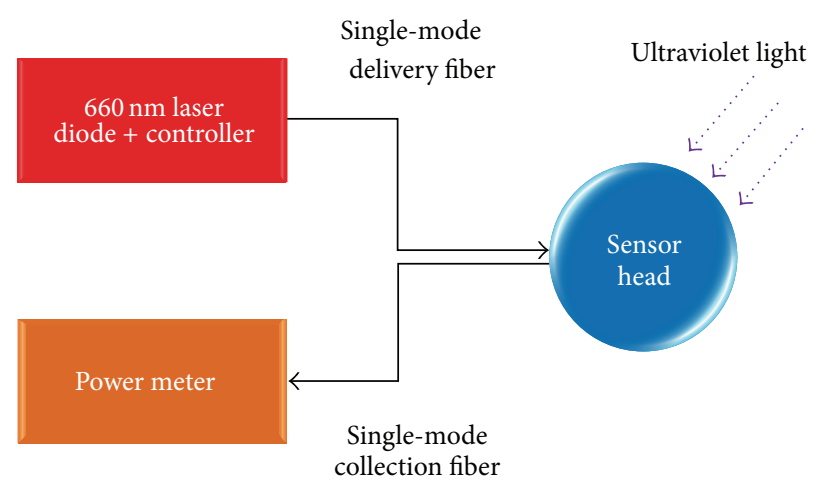

FIGURE 2: Schematic of the experimental setup.

(i.e., cuts above $400 \mathrm{~nm}$ wavelength) measuring $1 \mathrm{~cm}$ in diameter and $3 \mathrm{~mm}$ in height (i.e., $1 \mathrm{~mm}$ layer thickness), which can be readily obtained from commercial off-the-shelf stock. This filters out visible light from the ambient environment to minimize noise and thus enhance the sensitivity.

The second stage involves the fabrication of the photochromic preform (i.e., small or no core, cladding-doped) before it is pulled into fiber form using a conventional drawing tower [6] shown in Figure 3. It is difficult to produce $\mathrm{OM}$ directly from the preform and thus the process is split into separate stages. ZBLAN glass is an ideal candidate due to its low transition temperature (i.e., $260^{\circ} \mathrm{C}$ ), low intrinsic loss (i.e., $\sim 1 \mathrm{~dB} / \mathrm{km}$ ), and wide transparency window (i.e., $0.22-$ $8.0 \mu \mathrm{m}$ ) [7]. The minimum temperature of photochromic dyes can go below $-70^{\circ} \mathrm{C}$ [8]. Organic photochromic dyes such as spirooxazines and naphthopyrans have a high breakdown temperature of $300^{\circ} \mathrm{C}$ and thus can survive being added to a melt of lower transition temperature. They can be rapidly switched (e.g., activated within $50 \mathrm{~s}$ and deactivated within $15 \mathrm{~s}$ ) [9], and their transmission has a temperature dependence as low as $0.82 \% /{ }^{\circ} \mathrm{C}[10]$.

The third stage involves the fabrication of the OM [5] via the modified flame-brushing technique shown in Figure 3 [11]. This consists of heating the central section of the photochromic optical fiber while stretching the two ends to form a biconical taper whose uniform waist is the OM. The resulting $\mathrm{OM}$ has smooth and near-lossless transitions to regular-sized optical fibers, thus providing high connectivity with other fiberized components. 

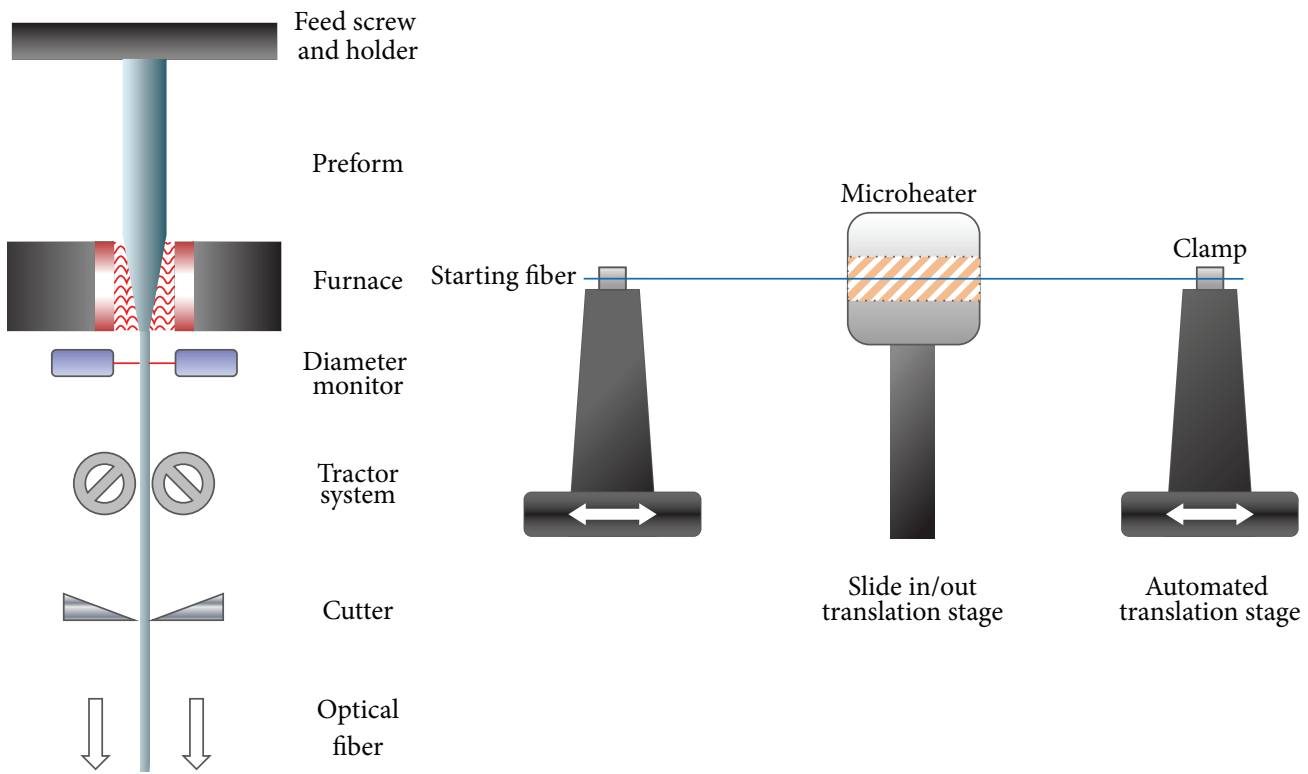

FIGURE 3: Schematic of the experimental setup.

The fourth stage involves spirally wounding a single layer of $\mathrm{OM}$ onto the surface using an automated rotation and translation rig to achieve a densely packed arrangement, as shown in Figure 4. The minimum bend radius can be in the order of millimetres due to the small diameter and high numerical aperture resulting from the large RI difference along the core-cladding interface. A single layer is the most efficient because the front-facing layer will absorb most of the UV light and leave little for the layers behind it. Before the coiling process, a thin layer (e.g., $>20 \mathrm{um}$ ) of low-RI polymer (e.g., Efiron PC-375) must be deposited on the disc to provide strong optical confinement and an adhesive surface for OM bonding. Each part of the OM is UV cured immediately after it comes in contact with the initial layer of polymer on the disc surface during winding. Then, a final layer of polymer is deposited and UV cured. This approach minimizes polymer shrinkage-induced changes to the OM layout in addition to ensuring high geometrical stability in the long term. The $\mathrm{RI}$ of the photochromic $\mathrm{OM}$ and polymer cladding are 1.50 and 1.38 , respectively, at a wavelength of $660 \mathrm{~nm}[7,12]$. The photochromic effect is expected to have a negligible impact on the RI of the OM [13].

The fifth and final stage involves sealing the glass-disc enclosure with a lid and housing the OM pigtails inside glass capillaries for high robustness. The resulting sensor head is highly robust to physical perturbations and chemical damage.

To clarify the manufacturing process, Figure 5 summarizes the various steps for fabricating the OM-based UV sensor.

\section{Results and Discussion}

To begin with, it is important to know the OM diameter will impact the maximum OM length that can be packed

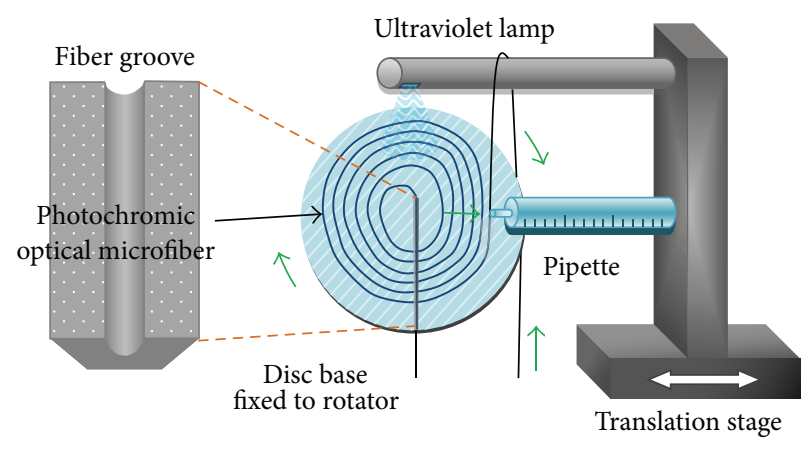

FIgURE 4: Schematic of the winding rig.

onto a disc of fixed size. The winding pitch is chosen to be equal to the mode-field diameter (MFD), so that negligible coupling occurs between adjacent windings. As seen from Figure 6 the optical confinement of OMs decreases the MFD with decreasing OM diameter [5]. However, below a diameter of $0.7 \mu \mathrm{m}$ (i.e., single-mode guidance), the diffraction effect overpowers the focusing effect of the waveguide and the MFD increases rapidly. Hence, the highest OM-packing density occurs at an OM diameter between 0.7 and $0.8 \mu \mathrm{m}$. Owing to the small OM diameter relative to the bend radii and its large numerical aperture, bend-induced stresses will have a negligible effect on the MFD.

The calculation of transmission uses the zero-UV received power as the reference, so that the responsivity is not influenced by the propagation losses but by the photochromic-induced loss. A visible-light transmission of $90 \% / \mathrm{cm}$ over a distance of $1 \mathrm{~cm}$ with an UV-A intensity of $15 \mathrm{~mW} / \mathrm{cm}^{2}$ and negligible propagation losses is highly feasible, because the photochromic-induced loss is by far 


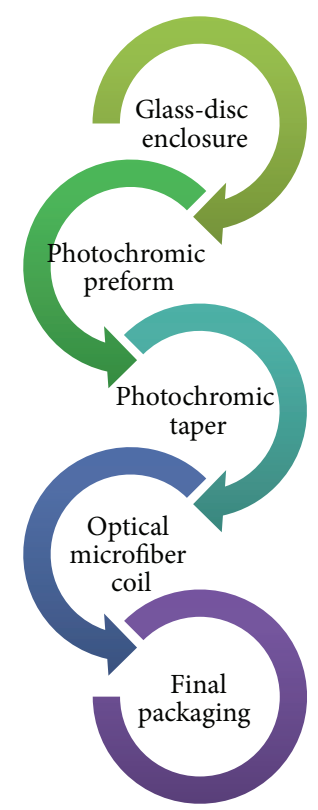

FIGURE 5: Process chart of sensor fabrication.

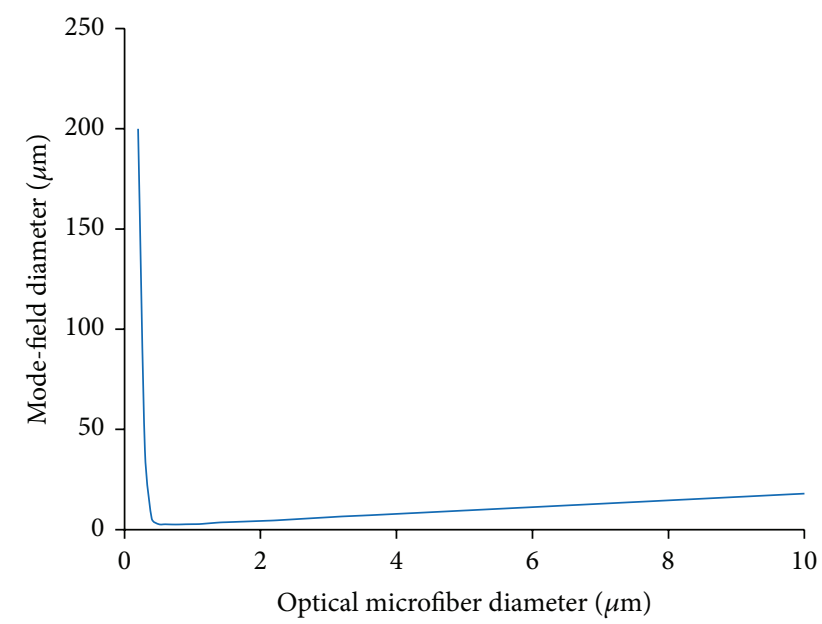

FIGURE 6: Relationship between the optical-microfiber diameter and the mode-field diameter. The core and cladding RIs are 1.50 and 1.38 at a wavelength of $660 \mathrm{~nm}$.

weaker than that in [14] (i.e., $42 \% / 5.5 \mu \mathrm{m}$ ). For the sake of simplicity, we assume that the photochromic dye is made in such a way that the transmission decreases linearly with increasing UV intensity. Therefore, the transmission can be expressed as

$$
T=\left(1-0.1 \times \frac{I_{\mathrm{UV}}}{15}\right)^{L}
$$

where $I_{\mathrm{UV}}$ is the UV intensity in $\mathrm{mW} / \mathrm{cm}^{2}, L=\pi r^{2} / \mathrm{MFD}$ is the OM length in $\mathrm{cm}, r$ is the disc radius in $\mathrm{cm}$, and MFD is also given in $\mathrm{cm}$.

To understand the derivation of $(1),(1-0.1)$ indicates a drop in transmission of $10 \%$. Including $\left(I_{\mathrm{UV}} / 15\right)$ means for every multiple of $15 \mathrm{~mW} / \mathrm{cm}^{2}$, an additional $10 \%$ is removed.
Since optical loss is multiplicative, $L$ scales the transmission for multiples of one centimeter.

The responsivity (i.e., optical response per measurand) is calculated by dividing the change in transmission $(\Delta T$ with units of $\mathrm{dB}$ ) by the UV intensity (i.e., units of $\mathrm{W} / \mathrm{cm}^{2}$ ). A high responsivity (i.e., high value) is desirable because it allows the sensor to detect a weak UV signal:

$$
R=\frac{\Delta T}{I_{\mathrm{UV}}}
$$

The UV sensitivity (i.e., detection limit of measurand) is calculated by dividing the transmission sensitivity by the responsivity. A high sensitivity (i.e., low value) is desirable because it enables the sensor to detect a weak UV signal. The expression is intuitive in that when $T_{\text {noise }}$ is smaller, there is less noise and thus a lower $S$ is feasible. When $I_{\mathrm{UV}}$ is lower with the same $\Delta T$, a weaker measurand signal can be detected and thus a lower $S$ is obtainable. When $\Delta T$ is larger for the same $I_{\mathrm{UV}}$, scaling down can produce the same $\Delta T$ with a lower $I_{\mathrm{UV}}$. Hence, a weaker measurand signal can be detected, leading to a lower $S$ :

$$
S=\frac{T_{\text {noise }}}{R}=\frac{T_{\text {noise }} I_{\mathrm{UV}}}{\Delta T} .
$$

To envisage the performance of different sensor head configurations, Figure 7 reveals the responsivity influenced by disc radius and $\mathrm{OM}$ diameter. The contour pattern is similar to that of Figure 6 because the MFD determines the OMpacking density, which influences the OM length, $\Delta T$, and consequently the responsivity. It is clear that the responsivity is higher for smaller OM diameters, but not too small (e.g., $<0.7 \mu \mathrm{m}$ ), and larger disc radii. For disc radii ranging from 0.1 to $3 \mathrm{~cm}$ and $\mathrm{OM}$ diameters ranging from 0.2 to $10 \mu \mathrm{m}$, the maximum responsivity is $-3.32 \times 10^{6} \mathrm{~dB} /\left(\mathrm{W} / \mathrm{cm}^{2}\right)$, occurring at a disc radius of $3 \mathrm{~cm}$ and an OM diameter of $0.7-0.8 \mu \mathrm{m}$.

For a zero-UV received power of $1.00 \mathrm{~mW}$, detector noise of $-85 \mathrm{dBm}$ or $3.16 \mathrm{nW}$ (e.g., thermal noise, $1 / \mathrm{f}$ noise, and shot noise), and laser intensity noise of $\pm 0.1 \%$ or $1.00 \mu \mathrm{W}$, the system noise is $1.00 \mu \mathrm{W}$ and the transmission sensitivity is $-4.36 \times 10^{-3} \mathrm{~dB}$.

The UV sensitivity for various disc radii and OM diameters is given in Figure 8. The UV sensitivity is superior for smaller OM diameters, but not too small (e.g., $<0.7 \mu \mathrm{m}$ ), and larger disc radii. For disc radii ranging from 0.1 to $3 \mathrm{~cm}$ and OM diameters ranging from 0.2 to $10 \mu \mathrm{m}$, the highest UV sensitivity is $1.31 \mathrm{nW} / \mathrm{cm}^{2}$, occurring at a disc radius of $3 \mathrm{~cm}$ and an OM diameter of $0.7-0.8 \mu \mathrm{m}$.

From a reverse point of view, the maximum UV intensity that can be accepted by the sensor head is predicted in Figure 9. Higher intensities would drag the received power of visible light below the system noise, thus becoming undetectable. It is evident that whenever the sensor head is inferior at detecting UV light, the absorption of visible light is weak and thus a higher UV intensity is needed to bring the sensor to its lower detection limit. For disc radii ranging from 0.1 to $3 \mathrm{~cm}$ and $\mathrm{OM}$ diameters ranging from 0.2 to $10 \mu \mathrm{m}$, the highest maximum UV intensity is $148.15 \mathrm{~mW} / \mathrm{cm}^{2}$, occurring at a disc radius of $0.1 \mathrm{~cm}$ and an $\mathrm{OM}$ diameter of $0.2 \mu \mathrm{m}$. 


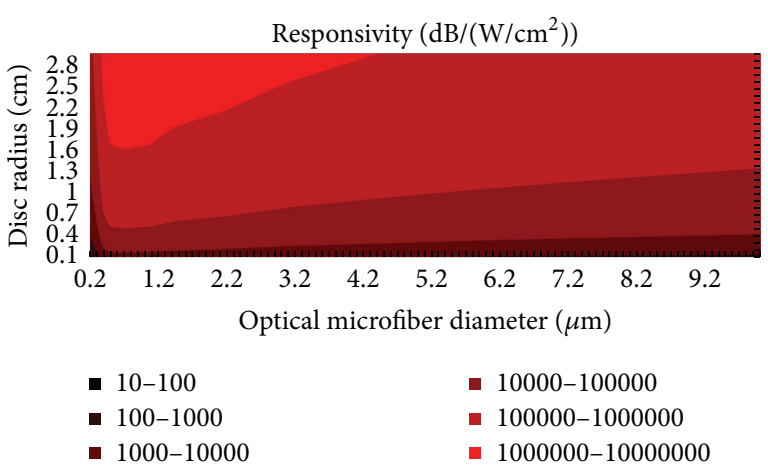

Figure 7: Dependence of responsivity (i.e., negative) on the disc radius and optical-microfiber diameter. The refractive index of the core and cladding is 1.50 and 1.38 at a wavelength of $660 \mathrm{~nm}$. The UV intensity is $15 \mathrm{~mW} / \mathrm{cm}^{2}$.

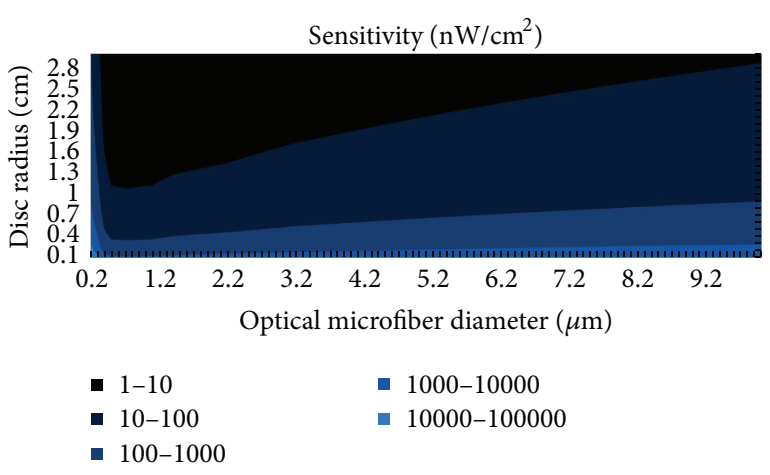

FIGURE 8: Dependence of sensitivity on the disc radius and opticalmicrofiber diameter. The refractive index of the core and cladding is 1.50 and 1.38 at a wavelength of $660 \mathrm{~nm}$. The UV intensity is $15 \mathrm{~mW} / \mathrm{cm}^{2}$.

Owing to the inherent trade-off, it is impossible for a sensor head configuration to exhibit both the lowestvalue UV sensitivity and the highest-value maximum UV intensity (i.e., high dynamic range). Hence, the nominal design of $1 \mu \mathrm{m}$ OM diameter and $2 \mathrm{~cm}$ disc radius (i.e., $\sim$ $457 \mathrm{~m}$ OM length) is chosen to deliver a good balance of UV sensitivity (i.e., $3.13 \mathrm{nW} / \mathrm{cm}^{2}$ ), maximum $\mathrm{UV}$ intensity (i.e., $22.66 \mu \mathrm{W} / \mathrm{cm}^{2}$ is high enough to detect harmful UV index of $9+$ ) and ease of fabrication (i.e., below $1 \mu \mathrm{m}$ is difficult to handle). The responsivity of $-1.39 \times 10^{6} \mathrm{~dB} /\left(\mathrm{W} / \mathrm{cm}^{2}\right)$ is illustrated in Figure 10 for this sensor head configuration, which can be used to aid the translation of observed changes in transmission into UV intensity. The trend is approximately linear for low UV intensities and for the linear regime the responsivity is slightly lower at $-1.32 \times 10^{6} \mathrm{~dB} /\left(\mathrm{W} / \mathrm{cm}^{2}\right)$. The accuracy of the UV sensor is the same as the UV sensitivity, which is $\pm 3.13 \mathrm{nW} / \mathrm{cm}^{2}$.

It is not possible to facilitate a comparison between the photochromic OM-based UV sensor and conventional fiber-optic counterparts due to the lack of parameters in the literature. However, traditional electrical counterparts exhibit sensitivities up to $104.11 \mathrm{nW} / \mathrm{cm}^{2}$ [15], and thus the

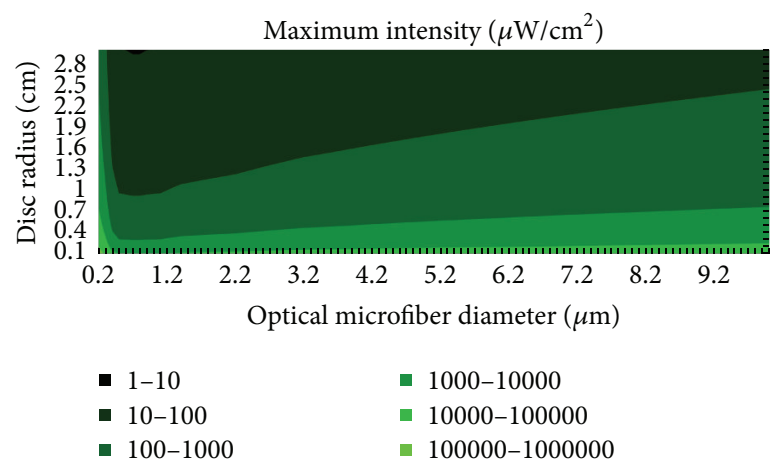

FIGURE 9: Dependence of the maximum ultraviolet intensity on the disc radius and optical-microfiber diameter. The refractive index of the core and cladding is 1.50 and 1.38 at a wavelength of $660 \mathrm{~nm}$.

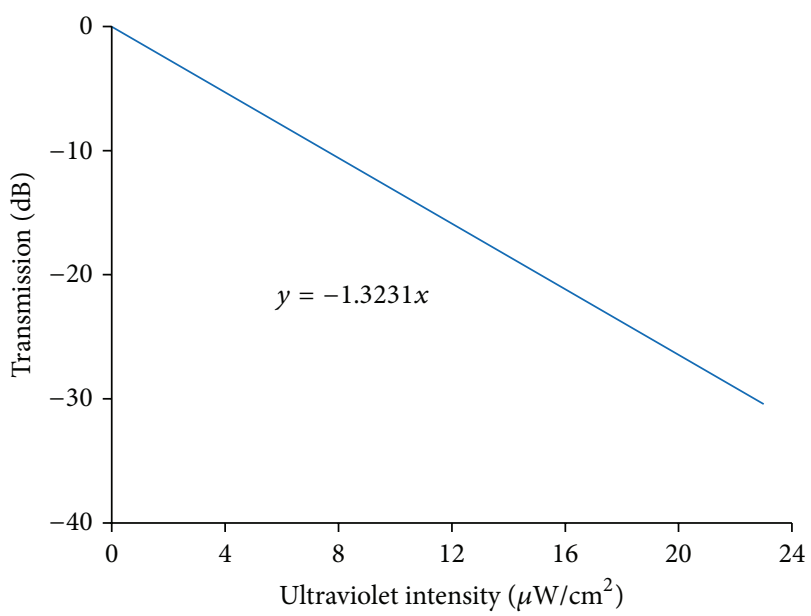

FIGURE 10: Relationship between the ultraviolet intensity and the transmission of the nominal sensor head configuration. The core and cladding RIs are 1.50 and 1.38 at a wavelength of $660 \mathrm{~nm}$.

performance enhancement of the OM-based design is at least one order of magnitude.

There is no fundamental limit to the detection bandwidth of the sensing mechanism, and thus the limiting factor resides with the power meter. Data sampling rates can be as high as $20 \mathrm{~Hz}$, which implies a detection bandwidth of $\sim 10 \mathrm{~Hz}$.

Although most photochromic dyes take tens of seconds [9] to fully respond to changes in the incident UV light, sensors based on such dyes are able to provide an early feedback before the steady state response is reached. For the application of monitoring the UV exposure of a person in a tanning booth or on the beach, a slow response (e.g., $>10 \mathrm{~s}$ ) is adequate because harmful effects only arise from longduration exposures. For the application of fire detection, a long response time (e.g., $>5 \mathrm{~s}$ ) is actually desired because it can filter out short spikes of UV light from sunlight reflections from moving objects that may trigger false alarms.

As previously mentioned, existing photochromic dyes including spirooxazines and naphthopyrans have a temperature range from -70 to $300^{\circ} \mathrm{C}[7,8]$. With advances 
in chemistry, future dyes are likely to have an extended operating range.

The transmission stability of some photochromic dyes can be within $0.82 \% /{ }^{\circ} \mathrm{C}[10]$. This is highly attractive for sensors based on such dyes due to the stable operation. For example, ambient temperature drifts of several degrees would only incur minor errors in the sensor output, and it is unlikely to trigger false alarms in the case of fire detection.

The sensor head is not designed to be selective with the incident UV light but instead to measure 3-dimensional UV signals from 3-dimensional space. Therefore, high crosssensitivities in each of the axes are desirable. Due to the discshape arrangement of OM (i.e., rather than a sphere), there is less detection area (i.e., Lambert's cosine law) for UV light from higher incident angles to the normal of the disc. As a result, the cross-sensitivities vary from high to low depending on the incident angle.

The size of the sensor head can be minimized by increasing the doping concentration of the photochromic dye, such that a shorter length of $\mathrm{OM}$ is needed to achieve the same responsivity. The lower limit of compactness is set by the size of the glass capillaries. Owing to the high compactness, lack of electrical terminals, and dielectric-material packaging, the UV sensor would exhibit low intrusiveness in any environment it is deployed in. Materials such as glass and low-density polymer are not heavy in small quantities. Therefore, the compact UV sensor is likely to weigh less than $9 \mathrm{~g}$ assuming the density of ZBLAN glass is similar to ordinary glass (i.e., $\left.2400 \mathrm{~kg} / \mathrm{m}^{3}\right)$ [16].

A high portability can be expected from the UV sensor, because of its simplistic hardware requirements. For the light source, a compact laser diode with a controller is sufficient. For the light detector, a nonbulky integrating sphere with a power meter is adequate. The ease-of-positioning can be regarded as easy, as the $\mathrm{OM}$ is well packaged in a robust glass-disc enclosure that can be mounted anywhere, even in hazardous environments. Due to the small size of the sensor head (i.e., several centimeters), it is generally not considered intrusive.

The intrinsic propagation loss of ZBLAN glass is known to be $\sim 1 \mathrm{~dB} / \mathrm{km}$ at $660 \mathrm{~nm}$ wavelength [7]. This is one order of magnitude lower than that of silica at $\sim 10 \mathrm{~dB} / \mathrm{km}$ [7].

The repeatability of successive measurements under the same conditions is expected to be high, as the transmission stability is excellent for high-quality samples of photochromic dye. On the contrary, the lifespan of the sensor head would depend on the degradation rate of the polymer cladding. Polymers such as the Efiron PC-375 are far more stable than Efiron PC-373 from experience, due to their different chemical compositions. Generally, the degradation is caused by postcuring and shrinkage of water content from the material.

With automated techniques to minimized human errors during the fabrication of photochromic preforms, fiber tapers, and spirally wound OM, the UV sensor can be manufactured with high reproducibility.

Lastly, the UV sensor is highly practical because it is relatively straightforward for it to be transported from a laboratory to a real measurement environment. This is made possible because the size and weight of the components and instruments used are very manageable, while the experimental setup is robust to external effects due to the all-fiber configuration and the protection of fragile parts.

\section{Conclusions}

An extremely responsive ultraviolet-light sensor based on photochromic optical microfiber has been theoretically demonstrated as a novel and competitive alternative to existing technologies. For a disc of $2 \mathrm{~cm}$ radius packed with optical microfiber of $1 \mu \mathrm{m}$ diameter, the predicted responsivity and ultraviolet sensitivity are $-1.39 \times 10^{6} \mathrm{~dB} /\left(\mathrm{W} / \mathrm{cm}^{2}\right)$ and $3.13 \mathrm{nW} / \mathrm{cm}^{2}$, respectively. The maximum ultraviolet intensity is $22.66 \mu \mathrm{W} / \mathrm{cm}^{2}$. The detection bandwidth and response time are $\sim 10 \mathrm{~Hz}$ and in the order of $\sim 10 \mathrm{~s}$, respectively. This novel design delivers an improvement in sensitivity by at least one order of magnitude compared to traditional electrical counterparts. Increasing the optical-microfiber length or the doping concentration of the photochromic dye leads to a higher responsivity (i.e., higher value), higher ultraviolet sensitivity (i.e., lower value), and lower maximum ultraviolet intensity (i.e., lower value). The same performance can be maintained with a smaller disc size by using an increased doping concentration of the photochromic dye to compensate for the shorter length of optical microfiber. Although the sensor is purely conceptual at the present time, we predict that the inevitable advances in chemistry and fiber fabrication will soon make photochromic doping highly feasible.

\section{Disclosure}

George Y. Chen is a first author and Zilong Wang is a coauthor.

\section{Conflict of Interests}

The authors declare that there is no conflict of interests regarding the publication of this paper.

\section{References}

[1] C. Lee, F. Matsuno, Y. Hashimoto, H. Okada, K. Sawada, and A. Wakahara, "Intelligent ultraviolet sensor composed of GaNbased photodiode and N-channel metal oxide semiconductor Si-charge transfer type signal processor," Japanese Journal of Applied Physics, vol. 51, no. 4, Article ID 044101, 2012.

[2] C. Fitzpatrick, E. Lewis, A. Al-Shamma'a, I. Pandithas, J. Cullen, and J. Lucas, "An optical fiber sensor based on cladding photoluminescence for high power microwave plasma ultraviolet lamps used in water treatment," Optical Review, vol. 8, no. 6, pp. 459462, 2001.

[3] A. V. Joža, J. S. Bajić, D. Z. Stupar, M. P. Slankamenac, M. Jelić, and M. B. Živanov, "Simple and low-cost fiber-optic sensors for detection of UV radiation," Telfor Journal, vol. 4, no. 2, pp. 133137, 2012. 
[4] J. Zmojda, M. Kochanowicz, P. Miluski, and D. Dorosz, "Sidedetecting optical fiber doped with $\mathrm{Tb}^{3+}$ for ultraviolet sensor application," Fibers, vol. 2, no. 2, pp. 150-157, 2014.

[5] G. Y. Chen, M. Ding, T. P. Newson, and G. Brambilla, "A review of microfiber and nanofiber based optical sensors," The Open Optics Journal, vol. 7, pp. 32-57, 2013.

[6] Y.-C. Huang, J.-S. Wang, Y.-K. Lu et al., "Preform fabrication and fiber drawing of $300 \mathrm{~nm}$ broadband Cr-doped fibers," Optics Express, vol. 15, no. 22, pp. 14382-14388, 2007.

[7] D. Dooling, "ZBLAN continues to show promise," NASA Science News, 1998, http://science.nasa.gov/science-news/science-at-nasa/1998/msad05feb98_1.

[8] M. Irie, Y. Yokoyama, and T. Seki, New Frontiers in Photochromism, chapter 8, Springer, 2013.

[9] Reversacol photochromic dyes, 2014, http://www.medvancesolution.com/dyes.html.

[10] R. Pardo, M. Zayat, and D. Levy, “Temperature dependence of the photochromism of naphthopyrans in functionalized sol-gel thin films," Journal of Materials Chemistry, vol. 16, no. 18, pp. 1734-1740, 2006.

[11] G. Brambilla, "Optical fibre nanowires and microwires: a review," Journal of Optics, vol. 12, no. 4, Article ID 043001, 2010.

[12] Efiron Optical Fiber Coating Technical Data Sheet, 2014, http://www.docstoc.com/docs/26393482/EFIRON-Optical-Fiber-Coating 1 1_.

[13] M. Hoshino, F. Ebisawa, T. Yoshida, and K. Sukegawa, "Refractive index change in photochromic diarylethene derivatives and its application to optical switching devices," Journal of Photochemistry and Photobiology A: Chemistry, vol. 105, no. 1, pp. 75-81, 1997.

[14] C. Chang, C. Wang, Y. Chen, and L. Cheng, "Preparation and characterization of spironaphthooxazine/polyacrylate photochromic hard coatings on plastic substrates," Journal of Applied Science and Engineering, vol. 17, no. 2, pp. 167-174, 2014.

[15] A. Vasudevan, S. Jung, and T. Ji, "On the responsivity of UV detectors based on selectively grown $\mathrm{ZnO}$ nanorods," IEEE Sensors Journal, vol. 12, no. 5, pp. 1317-1325, 2012.

[16] Density of glass, 2014, http://hypertextbook.com/facts/2004/ ShayeStorm.shtml. 

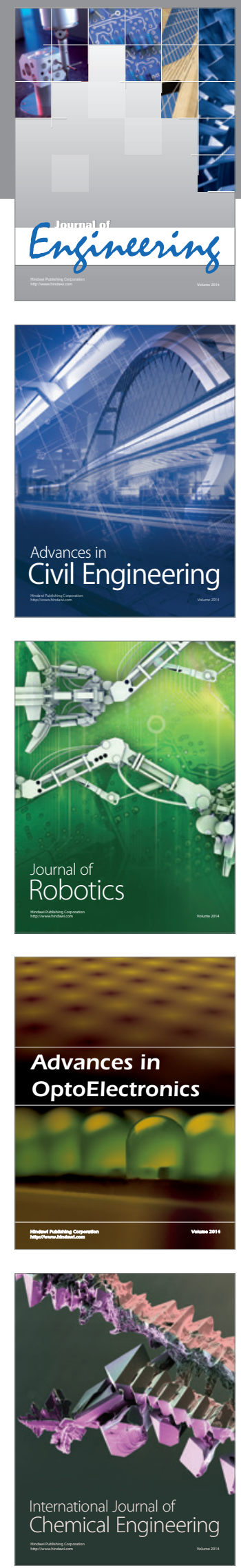

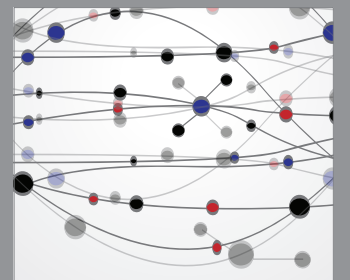

The Scientific World Journal
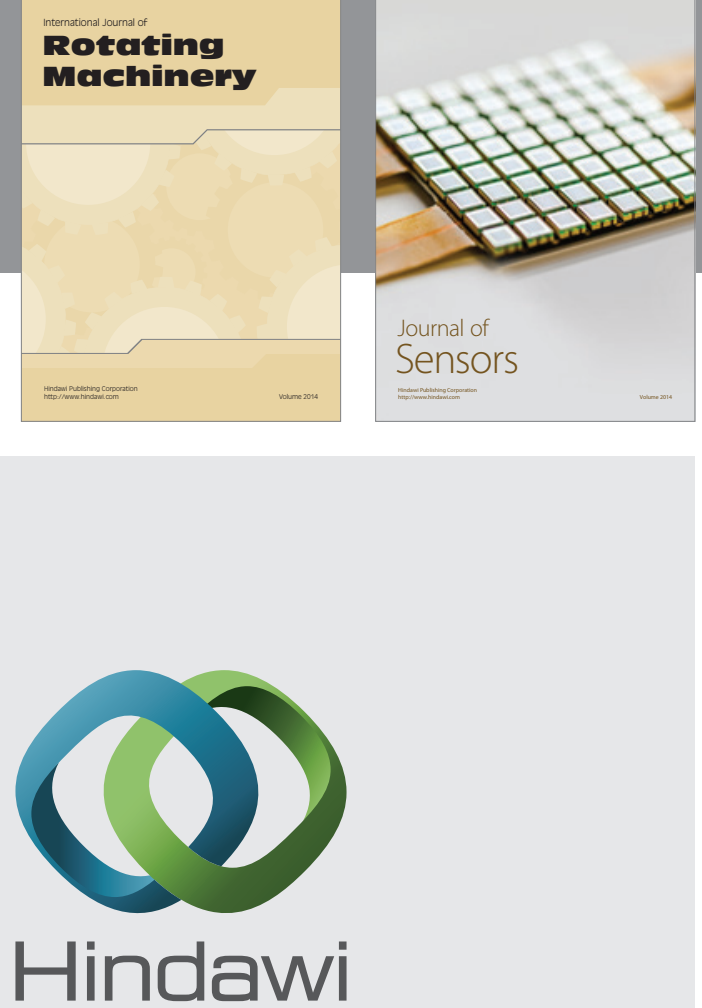

Submit your manuscripts at http://www.hindawi.com
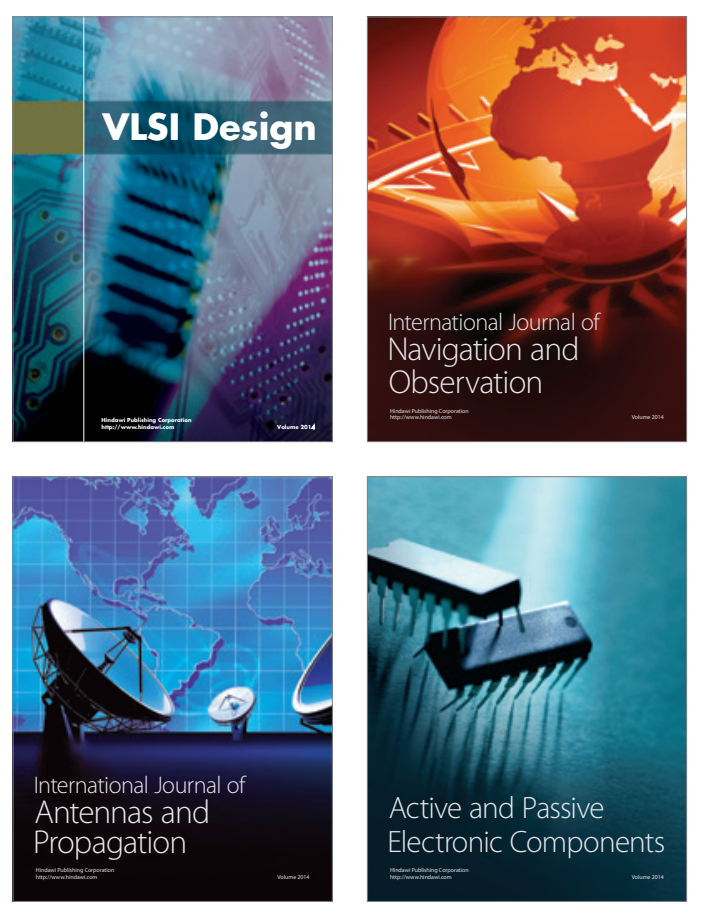
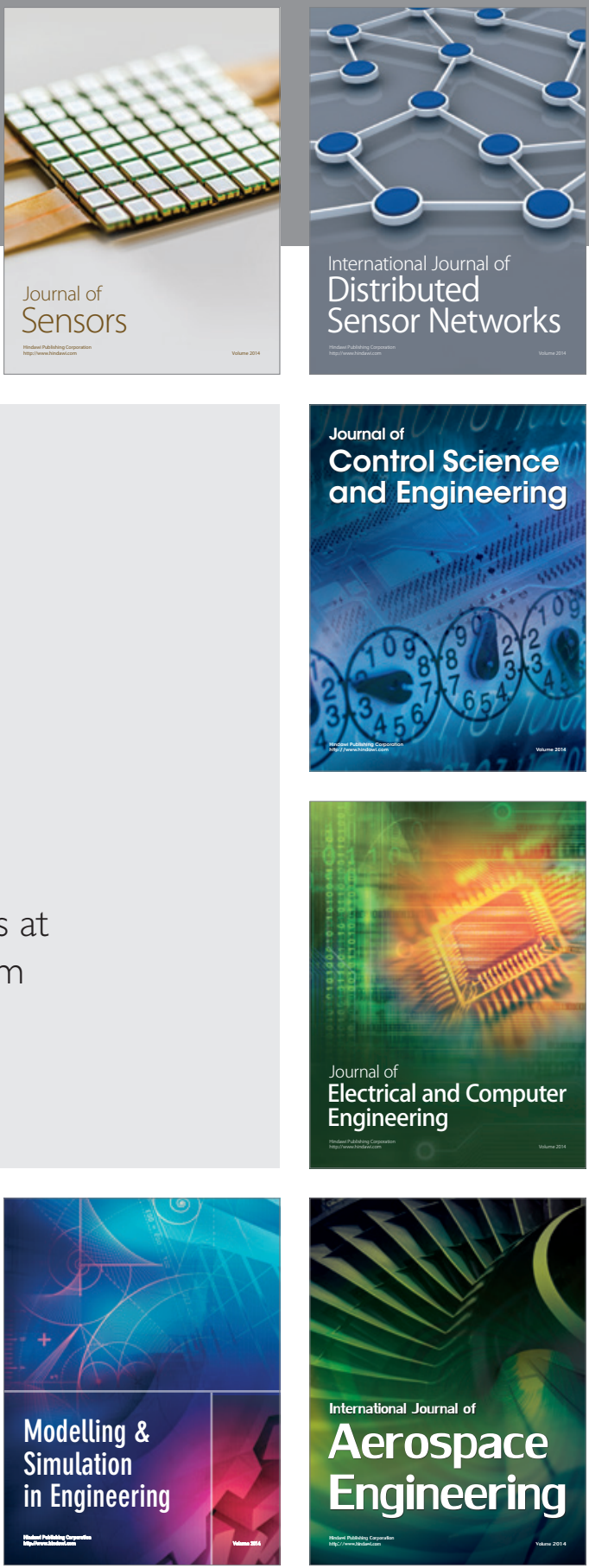

Journal of

Control Science

and Engineering
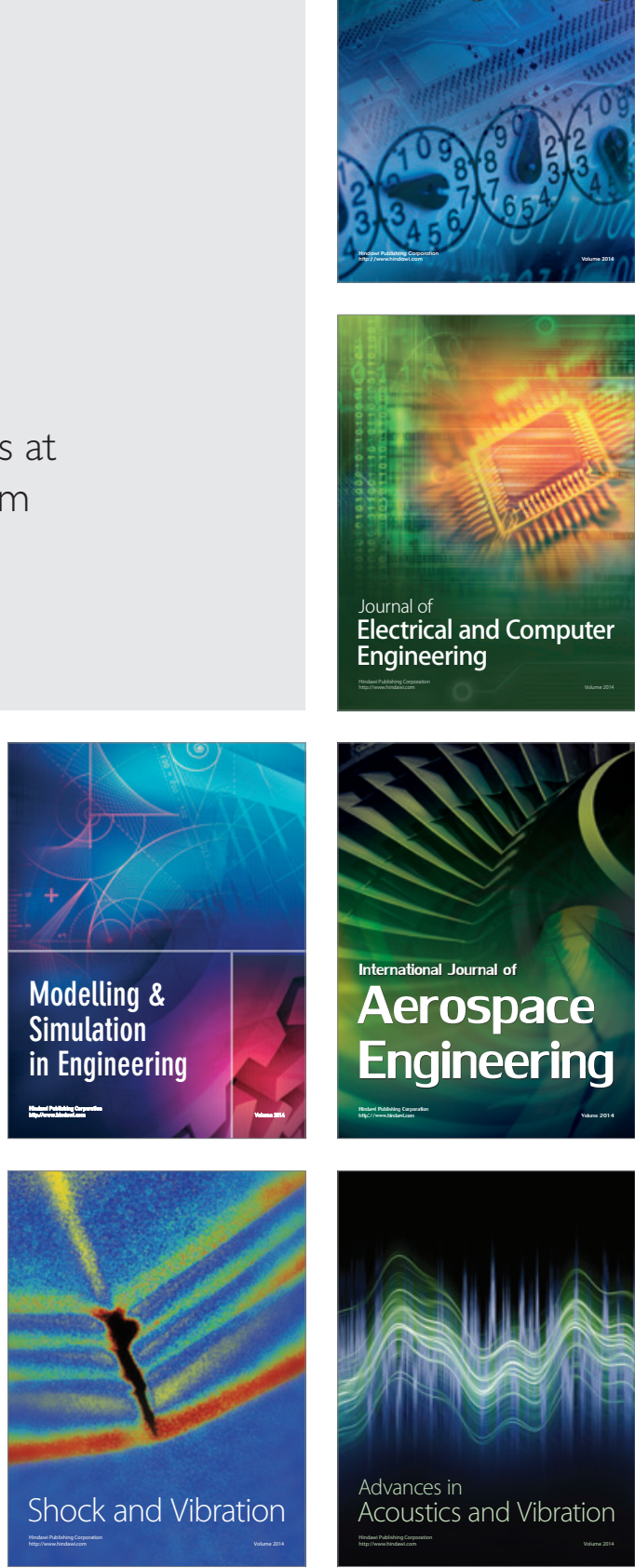